\title{
CONTAMINAÇÃO DO SOLO DO ESTADO DO RIO DE JANEIRO PELO C. TETANI. I - RELAÇÃO COM A DISTRIBUIÇÃO GEOGRÁFICA DO TÉTANO *
}

\author{
Walter Tavares $* *$ e Roched $\mathbf{A}$. Seba $* * *$
}

O tétano constitui um dos principais problemas de saúde Pública do Estado do Rio de Janeiro com o coeficiente de morbidade de 9,6/100.000 hab. A morbidade varia entre os diversos municipios do Estado, sendo maior em municípios do litoral e com baixa altitude, havendo menor morbidade em municipios localizados em zonas montanhosas.

Baseados em trabalhos realizados por autores estrangeiros e em trabalho previo que demonstram ser o $\mathrm{C}$. tetani isolado do solo e que há relação entre a morbidade do tétano e o grau de contaminação do solo, os autores colheram 608 amostras de solo de 60 municipios do Estado do Rio de Janeiro, durante a primavera e realizaram culturas e inoculação em camundongos. O C. tetani, foi demonstrado em 167 amostras $(27,4 \%$, sendo isolado de pastos, lavouras, ruas de cidades, estradas, etc. Embora o bacilo tenha sido isolado tanto em municipios de baixas altitudes como em zonas serranas, houve uma certa relação entre a altitude e o grau de contaminação do solo, sendo o bacilo isolado mais freqüentemente em municipios com baixa altitude.

Foi realizada uma análise da relação entre a morbidade do tétano e o grau de contaminação do solo em 60 municipios, sendo demonstrado que existe a relação, sendo maior a morbidade em regióes de maior contaminação de solo. Esta relação não foi absoluta pois outros fatôres influenciam na avaliação da morbidade da doença, como as condições sócio-econômicas da população, a precariedade de serviços médicos em alguns municipios e a notificação deficiente.

Em conclusão verifica-se que no Estado do Rio de Janeiro a distribuição geográfica do tétano não é uniforme, havendo certa relação com a contaminação do solo pelo $\mathrm{C}$. tetani, devendo-se estabelecer prioridade para imunização ativa da população contra o tétano nas regiōes com indices de morbidade mais elevados e nas que apresentam alto grau de contaminação do solo pelo bacilo tetânico.

* Trabvalho do Serviço de Doenças Infecciosas e Parasitárias do Hospital Universitário Antônio Pedro (Fac. Medicina U.F.F.) e do Instituto Vital Brazil S.A.. apresentado no VI Cong. da Soc. Bras. Medicina Tropical, Pôrto Alegre, 1970.

* Assistente da Disciplina de Clínica de Doencas Infecciosas e Parasitárias da Fac. Medicina U.F.F (Hospital Universitário Antônio Pedro).

*** Diretor Científico do Instituto Vital Brazil S.A.

Recebido para publicacão em 16-4-70. 


\section{INTRODUÇÃO}

Em estudo preliminar, realizado anteriormente (12), sôbre a contaminação do solo do Estado do Rio de Janeiro pelo $C$. tetani, observamos que em 55 amostras de solo colhidas em alguns municípios do Estado, houve positividade para o bacilo do tétano em $31 \%$ das amostras. Naquela oportunidade ressaltamos que os resultados apresentados demonstravam que o $C$. tetani é encontrado com freqüência no solo do Estado do Rio de Janeiro e nos propusemos a realizar estudo mais amplo, com maior número de amostras colhidas em vários municípios, a fim de avaliar a presença do C. tetani em todo o Estado e relacionar a morbidade da doença com a presença do bacilo tetânico no solo. Os resultados dêste estudo constituem esta comunicação.

\section{DISTRIBUIÇÃO GEOGRÁFICA DO TÉ- TANO NO ESTADO DO RIO DE JANEIRO}

De acôrdo com os dados recolhidos em fontes oficiais da Secretaria de Saúde do Estado do Rio de Janeiro $(1 ; 2)$ o tétano apresenta alta incidência no Estado, figurando em 6" lugar entre as doenças transmissiveis de maior ocorrência, com o índice de morbidade de 9,6/100.000 hab. no período de 1964/1966.

Os dados oficiais sôbre a ocorrência do tétano no Estado do Rio de Janeiro não refletem, porém, a verdadeira extensão e distribuição da doença. Em alguns municípios fluminenses a assistência médica é deficiente e a notificação da doença é realizada de modo precário, sendo possivel a ocorrência de casos de tétano sem que o Serviço de Estatística de Saúde tenha conhecimento. Por outro lado, o registro oficial dos casos era feito em relação ao município em que foi prestada assistência médica ao doente ou onde se registrou o óbito, o que muitas vêzes não correspondia ao local de origem do paciente. Podemos constatar êste fato quando comparamos alguns dados referidos no Boletim Informativo do Serviço de Estatística de Saúde (2) com os dados de nossa casuística, no Hospital Universitário Antônio Pedro (Niterói). Como exemplo, podemos observar que no municí- pio de Cabo Frio não foi registrado oficialmente nenhum caso de tétano em 1966, entretanto, no mesmo ano, no Hospital Antônio Pedro foram internados 9 pacientes procedentes dêste município. Esta mesma observação pode ser feita em relação aos municípios de $\mathbf{S}$. Gonçalo, Itaboraí, Saquarema e outros municípios próximos a Niterói, para onde são transferidos grande número de doentes, em decorrência de melhores condições de atendimento na capital do Estado. O mesmo fato ocorre em municípios próximos no Estado da Guanabara, para onde são drenados grande número de tetânicos.

Tendo em vista êstes diversos fatôres que contribuem para a incorreção do registro de casos de tétano, torna-se difícil a avaliação exata da morbidade da doença segundo os municípios fluminenses. Contudo, procuramos realizar um levantamento da distribuição geográfica do tétano no Estado do Rio de Janeiro, baseados nos casos notificados à Secretaria de Saúde do Estado e em observações colhidas no Hospital Antônio Pedro (Niterói) e no Hospital Francisco de Castro (G.B.) durante o período de 1966 a 1968 . Na tabela I apresentamos os índices de morbidade anual médio por 10.000 habitantes nos municípios fluminenses baseados nas informações recolhidas. Os dados aqui apresentados, se não correspondem à verdadeira ocorrência da doença no Estado, refletem, o mais aproximadamente possível, a distribuição do tétano no Estado do Rio de Janeiro.

Observamos na tabela I que a morbidade do tétano não é uniforme no Estado do Rio de Janeiro, havendo variações entre os 63 municipios em que é dividido o Estado. Tomando por base o indice de morbidade do tétano em todo o Estado -. aproximadamente $1 / 10.000$ hab. - convencionamos a existência de Zonas de alta endemicidade, correspondendo aos 19 municípios com morbidade superior a $1 / 10.000$ hab.; Zonas de média endemicidade, nos 17 municípios com morbidade entre $0,5 \mathrm{e}$ 1/10.000 hab. e Zonas de baixa endemicidade, correspondendo aos 27 municípios com morbidade inferior a 0,5/10.000 hab. ou nula (fig. I).

A convenção que estabelecemos é passível de criticas, pois em países de maior desenvolvimento os índices de $0,5 / 10.000$ hab. $(5 / 100.000$ hab.) são considerados de 
T A B L A I

INDICE DE MORBIDADE E GRAU DE CONTAMINAÇÃO DO SOLO NOS MUNICIPIOS DO ESTADO DO RIO DE JANEIRO

\section{MUNICÍPIO}

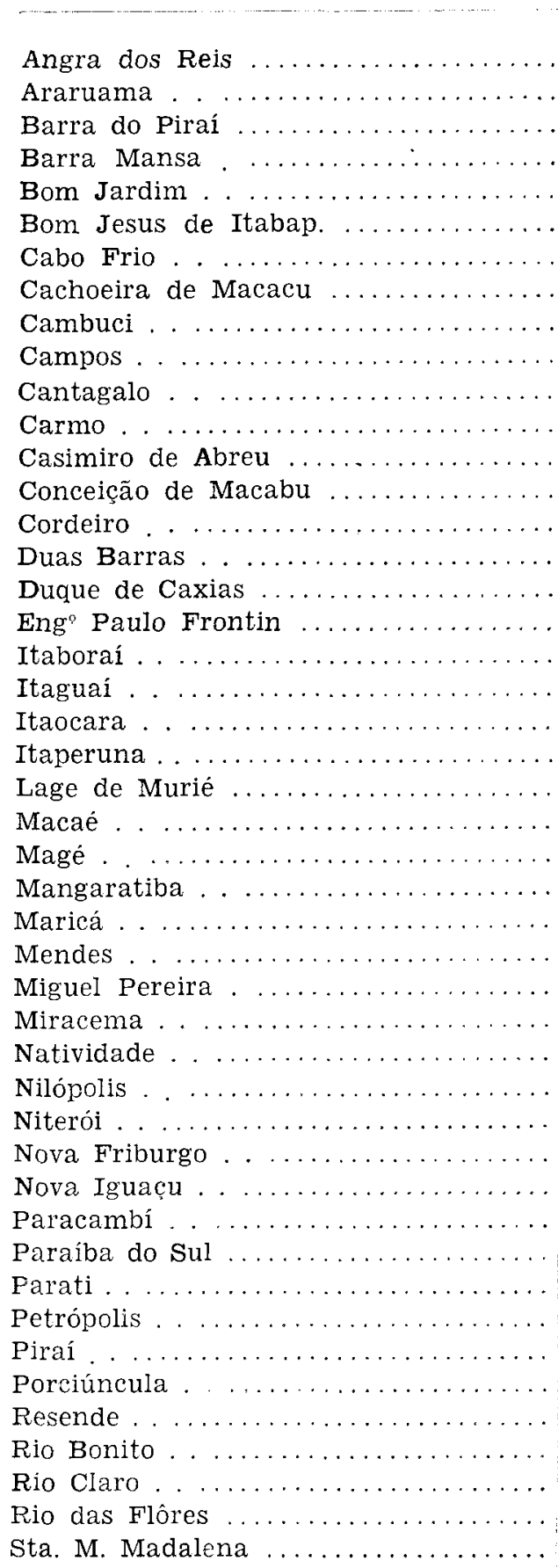

Indice de morbidade anual médio $\mathrm{P} / 10.000 \mathrm{hab}$.
Grau de contaminação pelo

C. tetani (\%)

\begin{tabular}{|c|c|}
\hline 1,59 & 30 \\
\hline 1,21 & 16,1 \\
\hline 0,66 & 30 \\
\hline 0,47 & 0 \\
\hline 0,4 & 30 \\
\hline 0 & 0 \\
\hline 3,27 & 30,5 \\
\hline 0,55 & 36,3 \\
\hline 0,48 & 42,8 \\
\hline 1,12 & 35 \\
\hline 0 & 21,4 \\
\hline 0,2 & 23 \\
\hline 0 & 9 \\
\hline 0,77 & 20 \\
\hline 0,7 & 38,4 \\
\hline 0 & 22,2 \\
\hline 1,98 & 30 \\
\hline 0,2 & 33,3 \\
\hline 1,45 & 30 \\
\hline 1,74 & 55,5 \\
\hline 0,33 & 27,2 \\
\hline 1,16 & 23 \\
\hline 0,2 & 37,5 \\
\hline 0,64 & 26,6 \\
\hline 1,39 & 50 \\
\hline 1 & - \\
\hline 0,76 & 10 \\
\hline 0,02 & 50 \\
\hline 0,5 & 25 \\
\hline 1 & 27,5 \\
\hline 0,3 & 11,1 \\
\hline 1,1 & 50 \\
\hline 1,64 & 70 \\
\hline 0,3 & 16,6 \\
\hline 1,54 & 45,4 \\
\hline 0,95 & 0 \\
\hline 0,58 & 37,5 \\
\hline 2,13 & - \\
\hline 0,35 & 15,3 \\
\hline 0,32 & 0 \\
\hline 0,49 & 30 \\
\hline 0,46 & 0 \\
\hline 0,54 & 20 \\
\hline 0 & 10 \\
\hline 0 & 14,2 \\
\hline 0,54 & 10 \\
\hline
\end{tabular}


TABELA I (Continuação da pág. anterior)

MUNICIPIO
Indice de morbidade anual médio

P 10.000 hab.
Grau de contaminação pelo

C. Tetani (\%)

Sto. A. Pádua

S. Fidelis . . . . . . . . . . . . . . .

0,71

0,79

0,82

0,13

1,16

1,13

0

0,45

1,54

0,2

0

0

0

0,5

0,52

0,18

0,82
10

45,4

33,3

0

57,1

31,2

0

0

45,4

33,3

-

0

37,5

50

62,5

40

Volta Redonda

OBS.: - Indices de morbidade calculados em relação aos dados de tétano ocorridos no período de 1966 1968 e em relação à população dos municípios estimada no Anuário Estatístico do Brasil, 1968.

alta morbidade $(3 ; 4)$. Acreditamos, entretanto, que no contexto da incidencia do tétano no Brasil, onde é atribuido o coef. de morbidade de 16/100.000 hab. (13), as regiōes com morbidade inferior a 0,5 10.000 possam ser classificadas como de menor endemicidade, devendo-se estabelecer prioridade para a imunização ativa contra o tétano nas regiões com indices superiores a êstes considerados.

Verificamos no Mapa I que a maioria dos municípios com alta endemicidade encontra-se próxima ao litoral, tornando-se menor a morbidade nos municípios do interior do Estado.

A distribuição geográfica do tétano não e uniforme em nenhum pais, sendo as regiões de clima quente, com solo fértil as que apresentam maior morbidade $(3 ; 4)$, caindo a incidência do tétano em regiōes de clima temperado ou frio.

Procuramos relacionar a distribuição desigual do tétano nos municípios do Estado do Rio de Janeiro com a altitude e o clima dos municípios. A topografia do Estado é muito acidentada pois é cortado pela Serra do Mar que se estende por vários municípios. Entretanto as altitudes observadas no Estado não são muito elevadas, sendo poucos os municípios que estejam com tôda sua área em região montanhosa ou acima de $400 \mathrm{~m}$. O clima do Estado é quente e úmido na maioria das regiōes, tornando-se temperado em zonas montanhosas.

$\mathrm{Na}$ tabela II relacionamos a distribuição geográfica do tétano com a altitude, considerando-se como limite a altitude de $400 \mathrm{~m}$, acima da qual o clima é geralmente temperado e ameno.

Verifica-se na tabela II que na maioria dos municípios com altitude superior a $400 \mathrm{~m}$ a morbidade pelo tétano foi menor, sendo maior nos municípios de baixa altitude. Foi realizada a análise estatística dêstes resultados com aplicação do teste de diferença de proporção e calculado o coeficiente de correlação linear, sen- 
T A BELA I I

RELAC̣ÃO ENTRE MORBIDADE POR TÉTANO E ALTITUDE DO MUNIĆ́PIO

Acima de $400 \mathrm{~m}$

Alta e média morbidade ......... - 5 municipios

Baixa morbidade . . ........... -16 municipios

Abaixo de $400 \mathrm{~m}$

Alta e média morbidade ......... 31 municipios

Baixa morbidade . . ............ 11 municípios

Análise estatistica:

$\begin{aligned} T & =3,75 \\ \text { Coef. correl. Linear } & =-0,544 \text { (forte) }\end{aligned}$

do confirmada a hipótese apresentada, ou seja, há uma relação entre a morbidade do tétano e a altitude, sendo maior a morbidade quanto menor a altitude. Entretanto, queremos ressalvar novamente que a notificação da doença é deficiente no Estado, sendo possivel que tal relação seja influenciada por êste fato.

\section{CONTAMINAÇÃO DO SOLO DO ESTADO DO RIO DE JANEIRO PELO C. TETANI}

Desde a descoberta do C. tetani, por Nicolaier, sabe-se que o bacilo do tétano é um habitante do solo, onde vive sob a forma de esporos. A partir desta descoberta vários pesquisadores, no estrangeiro, dedicaram-se ao estudo da contaminação do solo pelo bacilo, a fim de estabelecer a prevalência da bactéria em várias regiões, o tipo de solo em que ocorre maior prevalência, e as relações entre a morbidade da doença e o grau de contaminacáo do solo. Assim, Dubovsky e Meyer (7) em estudos sôbre a contaminação do solo dos Estados Unidos, estabeleceram que o $C$. tetani tem prevalência no Este e MeioOeste dos Estados Unidos, sendo encontrado não só em terras cultivadas como também em florestas virgens; Damon e Payabal (5), no Estado de Maryland, encontraram a positividade de $10,5 \%$ nas amostras estudadas; Gilles (8), estudando a ocorrência do $C$. tetani na poeira das ruas da cidade de Baltimore, encontra $14,2 \%$ de positividade, demonstrando as$\operatorname{sim}$ que o bacilo é encontrado mesmo em grandes cidades; Lavergne e col. (9), na França, encontram a positividade de $50 \%$ na região de Meuthe-et-Moselle, local onde o tétano apresenta alta incidência. Entretanto os trabalhos mais interessantes sôbre a contaminação do solo pelo $C$. tetani e as relaçôes com a morbidade da doença, foram realizadas por autores soviéticos, particularmente Sergeeva e Matveev $(10 ; 11)$ que estabeleceram que a morbidade da doença está diretamente relacionada com o grau de contaminação do solo, encontrando o bacilo em $25 \%$ das amostras colhidas na região do Krasnodar, local de alta incidência do tétano; $19 \%$ no Turkmem, onde a incidência é menor e sòmente $3 \%$ na região de Moscou onde é muito baixa a incidência do tétano.

Baseados nos trabalhos realizados por êstes autores estrangeiros e tendo em vista que o Estado do Rio de Janeiro apresenta alta incidência do tétano e que em estudo preliminar encontramos alto percentual de positividade para o bacilo tetânico em pequeno número de amostras de solo examinadas, procuramos verificar o grau de contaminação do solo nos vários municípios e relacionar a morbidade do tétano com a presenca de bacilo no solo. Para tanto, recolhemos 608 amostras de solo, durante a primavera, em 60 dos $63 \mathrm{mu}$ - 
ESTADO DO RIO DE JANEIRO

DIVISÄO MUNICIPAL

Escolo- 1400000

\section{INDICES DE MORBIDADE ANUAL MÉDIO POR}

MUNICIPIO POR 10.000 HABITANTES (Período

$|966-68|$

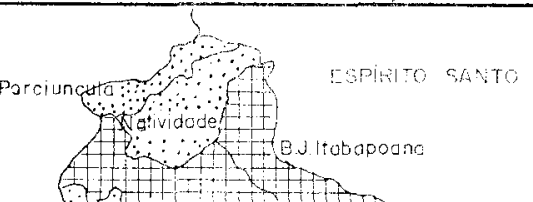

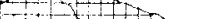

de dirnót $\therefore \therefore-1$.

Mition

Mijacerno

Tिint

S. A Paduaf

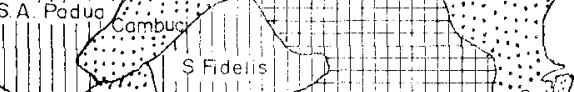

Le 5.S.Alto (s.) 1 li

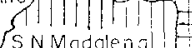

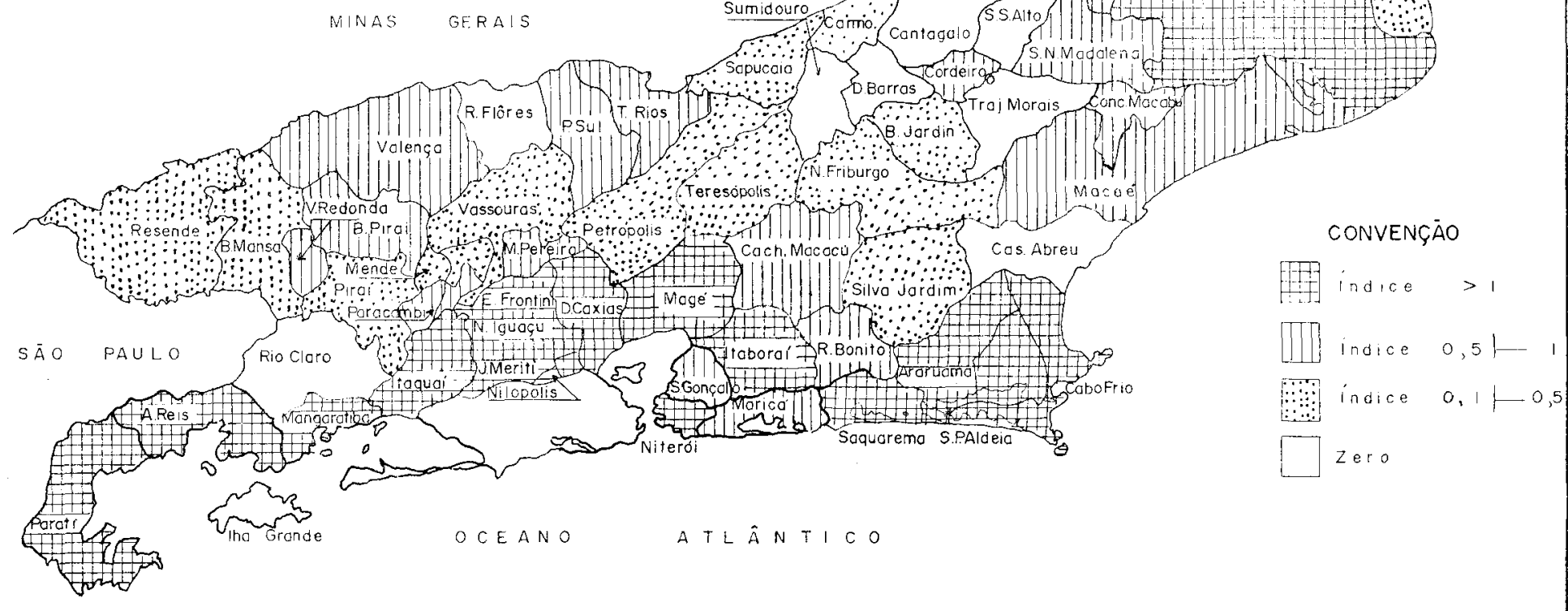


nicipios fluminenses, sendo colhidas em média 10 amostras de cada município. A terra foi coletada em frascos esterilizados, desde a superfície do solo até uma profundidade de 3 a $5 \mathrm{~cm}$. sendo colhidas amostras de pastos, lavouras, jardins, ruas de cidades, estradas, residencias, praias, etc. O material foi levado ao Instituto Vital Brazil onde foram realizadas as culturas e testes biológicos em camundongos para verificação do $C$. tetani. Para isso pesou-se $5 \mathrm{~g}$ da amostra de terra e dissolveu-se em $10 \mathrm{ml}$ de solução salina. Após agitação por 3 minutos deixou-se em repouso por 2 horas. Da solucão sobrenadante foi aspirado $0,5 \mathrm{ml}$ que foi semeada em meio de Tarozzi. Após 4 dias de culturas em anaerobiose o meio foi centrifugado e do sobrenadante aspirou-se $0,1 \mathrm{ml}$ que foi inoculado em camundongos por via I.M.., deixando-se os animais em observação por 4 dias. Nas amostras que apresentaram positividade para o $C$. tetani realizamos a prova de neutralização da toxina tetânica, pela injeção em novos camundongos do material de cultura em mistura com sôro anti-tetânico.

Verificamos que das 608 amostras houve positividade para o bacilo em 167 , isto é, $27,4 \%$ de amostras positivas, o que demonstra que o solo do Estado do Rio de Janeiro apresenta alta contaminação pelo bacilo tetânico, confirmando os dados apresentados em nosso estudo preliminar. A positividade nas amostras colhidas não foi uniforme entre os municípios estudazero como pode ser observado na tabela $I$. Baseados nestas diferenças entre municípios realizamos um mapeamento sôbre a distribuição do C. tetani no solo do Estado do Rio de Janeiro (Figura IT). Consideramos os municípios que apresentam contaminação superior a $30 \%$, que é aproximadamente a média cbtida em todo o Estado, como zonas de elevado grau de contaminação; os municípios entre $15 \mathrm{e}$ $30 \%$ como apresentando regular contaminação e os municípios com menos de $15 \%$ cu zero como de baixa contaminação. Verificamos por êste critério que a contaminação do solo do Estado foi alta ou regular em 43 municípios e houve baixa contaminação em 17 municípios. Chama a atenção que o local de maior positividade foi na Capital do Estado, a cidade de Niterói, onde colhemos o material em ruas de diversos bairros, residências, jardins públicos, encontrando-se o bacilo em $70 \%$ da amostra examinada.

Procuramos verificar se o grau de contaminação do solo dos municípios fluminenses estava relacionado com a altitude da região (tabela III) pois outros autôres referem menor presença de bacilos nas regiōes montanhosas.

Verificamos pela tabela III que o $C$. tetani foi isolado com freqüência não só em regiões de baixa altitude mas, também, em ronas montanhosas. Procedemos à análise estatística dêstes resultados para

T A B E L A I I I

RELAÇÃO ENTRE CONTAMINAC̣ÃO DO SOLO E ALTITUDE DO MUNICiPIO

\begin{abstract}
RELACAO ENTRE CONTAMINACAO DO SOLO E ALTITUDE DO MUNICIPIO
\end{abstract}
Acima de $400 \mathrm{~m} \quad$ Alta ou regular contaminação ....... 12 municípios

Baixa contaminação . . .......... 9 municípios

Abaixo de $400 \mathrm{~m} \quad$ Alta ou regular contaminação ...... 31 municipios

Baixa contaminação . . ........... 8 municípios

Análise estatística:

$$
\begin{aligned}
& \mathrm{T}=1,83 \\
& \mathbf{P}=0,0467
\end{aligned}
$$

Coef. correl. Linear $=0,326$ (regular fraco) 


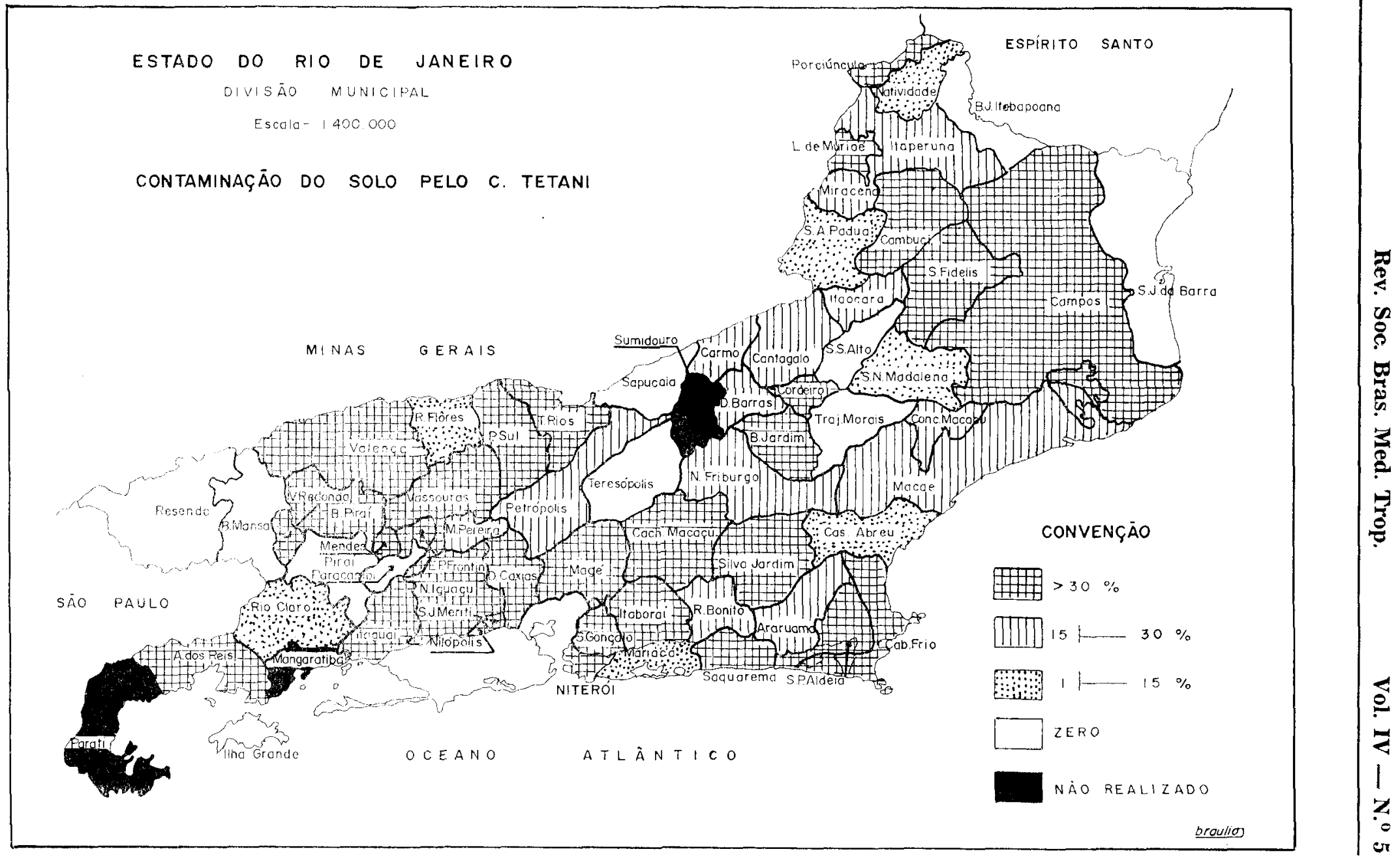


avaliar a importância de altitude na contaminaçāo do solo pelo bacilo. O teste de diferença de proporção e o teste exato de Fischer revelaram que a altitude na amostra examinada exerce papel influente, sendo o bacilo isolado mais freqüentemente em baixas altitudes que zonas montanhosas. Entretanto o coeficiente de correlação linear mostrou que esta relação não é absoluta, já que na amostra examinada o bacilo também foi encontrado em certa proporção em zonas montanhosas do Estado do Rio.

Em paises que apresentam regiōes de altas montanhas a relação entre a altitude e a contaminação do solo é mais evidente que no estudo por nós efetuado. Denchev (6), na Bulgária, refere que a contaminação é maior em planícies e menor em regiōes montanhosas; Sergeeva e Matveev (11), em extenso estudo na U.R.S.S., demonstram que o bacilo tetânico não é encontrado nas regiões montanhosas das províncias de Altai, onde o clima frio não favorece o crescimento do C. tetani no solo, fator que provàvelmente influencia o grau de contaminação do solo pelo bacilo. No Estado do Rio de Janeiro, situado em zona tropical. não se observa a influência climática importante dos paises e regiões frias, pois mesmo nas regiões serranas não existem elevadas altitudes e o clima é temperado, não influenciando o crescimento do $C$. tetani como provàvelmente o faz o clima frio.

Verificamos, por fim, a relaçāo entre a morbidade do tétano nos municipios flu- minenses e o grau de contaminação do solo (tabela IV). Para maior facilidade de exposição e conclusōes consideramos os municípios de regular e alta contaminação englobados como tendo alta contaminação assim como os de média e alta morbidade englobados como tendo alta morbidade.

Observa-se pela tabela IV que em nosso material houve relação entre a morbidade do tétano e o grau de contaminação do solo em $41 \quad(68,3 \%)$ dos municípios. Em 19 municípios não houve relação, sendo que em 14 houve alta contaminação do solo sem haver correspondência com a morbidade.

Procedemos à análise estatística dèstes resultados para verificar o valor desta conclusão. Utilizando o teste do quiquadrado, a análise demonstrou que há relação entre a morbidade do tétano e a contaminação do solo. O coeficiente de correlaçāo linear também comprova a relaçāo, porém não de modo absoluto.

É possivel que a ausência de correlaçāo absoluta entre morbidade do tétano e a contaminação do solo em nosso trabalho se deva à deficiência na notificação da doença. Observamos que muitos municipios em que houve alta contaminação e correspondente baixa morbidade são localizados em zona rural, com assistència médica precária, notificação deficiente, população com baixo nível sócio-econômico. sendo provável que ocorram vários casos de doença sem conhecimento oficial das autoridades sanitárias.

\title{
TABELA IV
}

\begin{abstract}
RELAÇAO ENTRE CONTAMINAC̣Ã DO SOLO E MORBIDADE POR TETANO NOS MUNICIPIOS DO ESTADO DO RIO DE JANEIRO
\end{abstract}

Houve relação - 68,3\%

Em 29 houve alta morbidade e alta contaminação

Em 12 houve baixa morbidade e baixa contaminacão

Em 5 houve baixa contaminação e alta morbidade

Não houve relação $-31,6 \%$

Em 14 houve alta contaminação e baixa morbidade

$x^{2}=3,84$

Coef. correl. Linear $=0,399$ (regular-forte). 


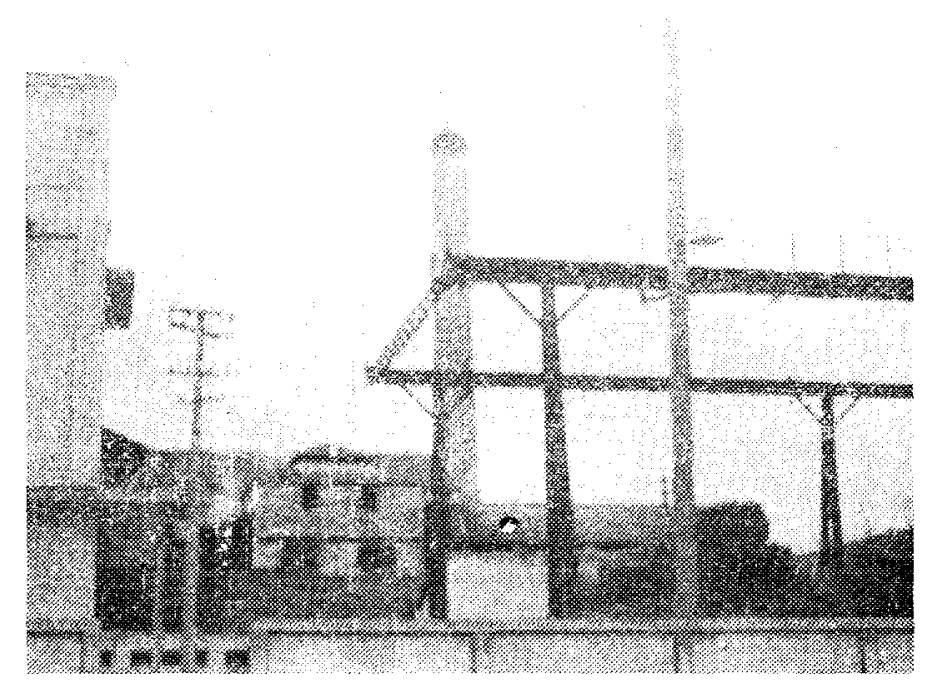
Fif. III - Campos. Usina de Açúcar. Cultura do solo
positiva para C. tetani

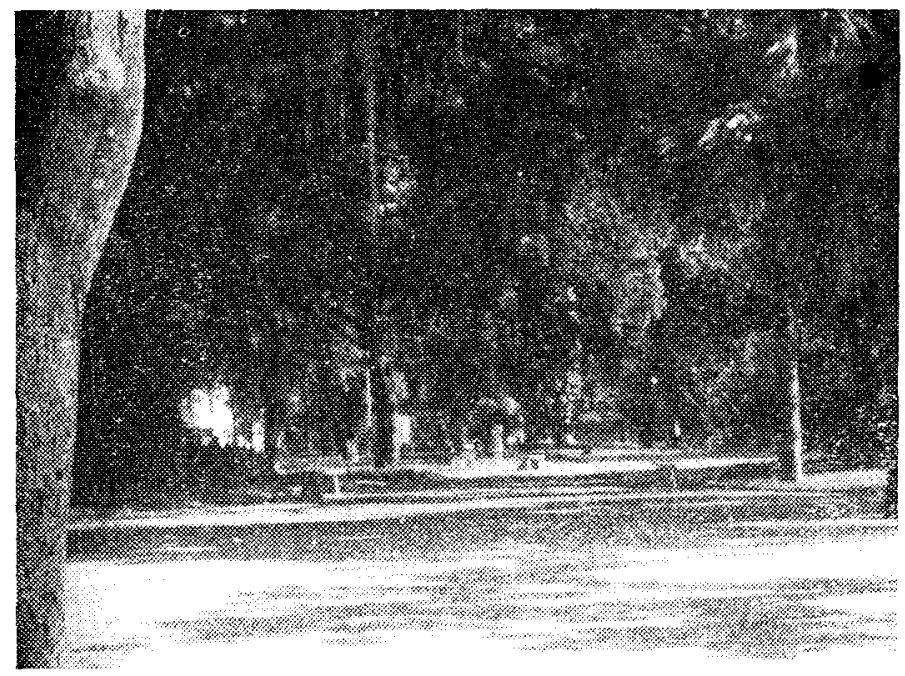

Fig. IV - Niterói - Jardim Público. Cultura do solo positiva para $C$. tetani 


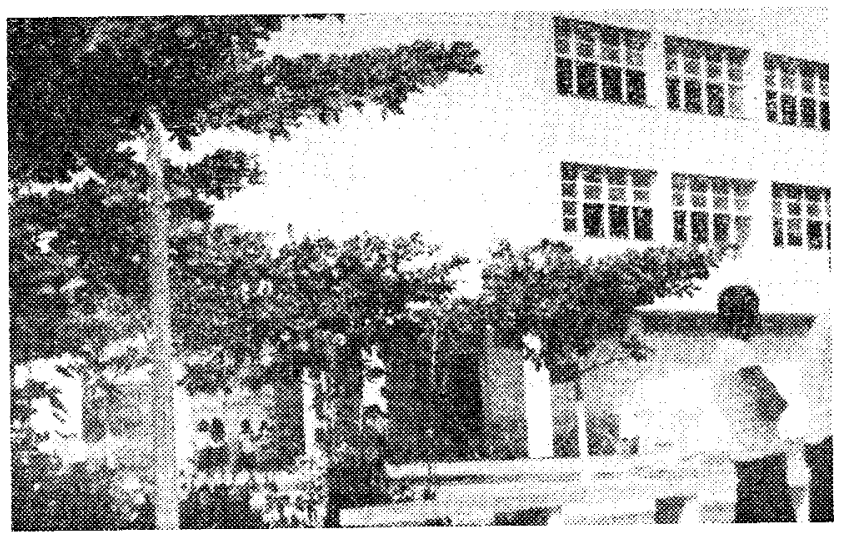

Fig. V - Miracema - Escola. Cultura do solo positiva para C. tetani

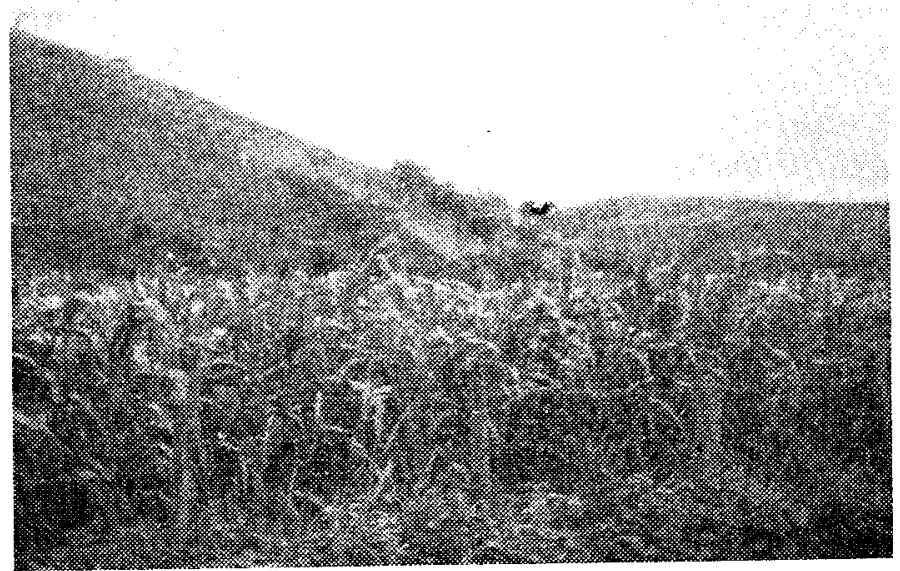

Fig. VI - São Fidelis - Lavoura de milho. Cultura do solo positiva para $C$. tetani 


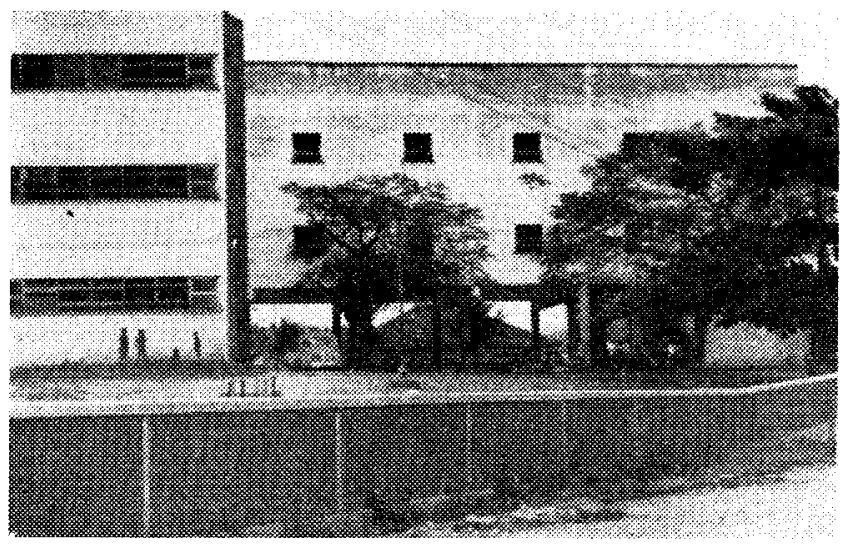

Fis. VII - Porciúncula - Grupo escolar. Cultura do solo positiva para $C$ tetani

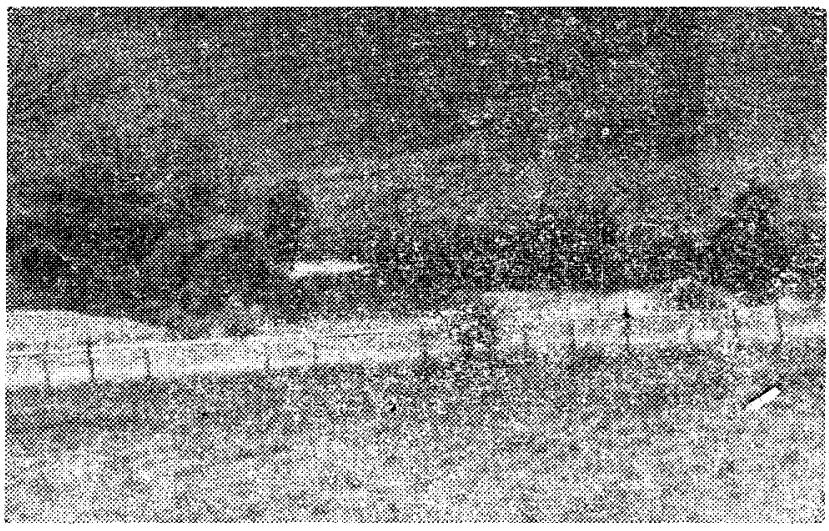

Fis. VIII - Itaperuna - Pasto. Cultura do solo positiva para $C$. tetani 


\section{AGRADECIMENTOS}

Os autores manifestam sua gratidão ao Sr. Elson C. Bittencourt pelo auxilio na realização das culturas e testes biológicos e ao Dr. Maurício de Pinho Gama do Departamento de Estatística da Fundação Ensino Especializado de Saúde Pública pela realização da análise estatística dêste trabalho.

\section{SUMMARY}

A serious Public Health problem in Rio de Janeiro State, tetanus in this State of Brazil presents an average morbidity coeficient of 9,6/100.000 inhabitants. The morbidity is different among the municipalities of the State, being greater in the municipalities of the litoral, in zones of low altitude.

Based on studies of soil contamination by C. tetani made by foreing authors and in a preliminary report, the authors colleted 608 soil samples from 60 municipalities of Rio de Janeiro State, during the spring, and made cultures and biological tests to investigate the presence of $\mathrm{C}$. tetani. Bacillus was found in 167 samples $(27,4 \%)$ collected in pastures, streets, beachs, gardens, etc. C. tetani was unequaly distributed through the state, being more frequent in anes of low altitude, but was found also in zones of temperated climate of the mountains.

A statistical analysis demonstrates the relationship between the morbidity of tetanus and the contamination of soil by $\mathrm{C}$. tetani in this state, but the relationship was not absolute in all municipalities.

\section{BIBLIOGRAFIA}

1 - Anuário do Serviço de Estatística de Saúde - Secretaria de Saúde e Assistência do Estado do Rio de Janeiro, $1966 / 67$.

2 - Boletim Informativo de Serviço de Estatística de Saúde - Outubro, 1969 - Secretaria de Saúde e Assistência do Estado do Rio de Janeiro.

3 - BYTCHENCKO, B. - Distribucion geografica mundial del tetanos. Revision del problema -- Bol. Ofic. Sanit. Panam., pag. 97, agôsto 1966.

4 - BYTCHENCKO, B. - Tetanus as a World problem - Proceedings of the International conference on tetanus, pag. 21, 1966 - Hans Huber Publishers, Bern and Stuttgart.

5 - DAMON, S. R. e PAYABAL, L. B. - Distribution of the spores of bacillus botulinus and bacillus tetani in the Soil -- J. Infec. Dis. 39: 491, 1926.

6 -- DENCHEV, V. - in Panel and Discussion on Epidemiology of tetanus - Proceedings of the International Conference on tetanus, pg. 99, 1966 - Hans Huber Publishers, Bern and Stuttgart.

77 - DUBOVSKY, B. J. e MEYER, K. F, - The ocurrence of $\mathbf{B}$. tetani in soil and vegetables - J. Inf. Dis. 31: 614, 1922 .
8-GILLES, E. C. - The isolation of Tetanus bacilli from street dust J.A.M.A. 109: 484, 1937.

9 - LAVERGNE, V.; HELlUY, J. R. e FAIVRE, G. - Contribution a l'étude morphologique et biologique du Clostridium tetani. Rev. Immunol. 13: 315,1949

10 - SERGEeVA, T. I. e MATVEEV, $\mathrm{K}$. I. - Tétano e sua profilaxia em tempo de paz - Trad. J. Hyg. Epid., Micro, e Imunol. 10: 133, 1966.

11 - SERGEEVA, T. I. e MATVEEV, $\mathrm{K}$. I. - Geografical distribution of $C$. tetani in the soil of the U.S.S.R. Proceedings of 'Intern. Conference on tetanus pag. 77, 1966 - Hans Huber Publishers, Bern. and stuttgart.

12 - TAVARES, W.; SEBA, R. A. e BITTENCOURT, E. C. - Estudo preliminar sôbre a contaminação do solo do Estado do Rio de Janeiro pelo C. tetani - Trabalho apresentado na 1. a Jornada de Microbiologia, Guanabara, 1959 - Em publicação.

13 - VERONESI, R. - Contribuição para o estudo clínico e experimental do tétano - Tese, 1960 , S. Paulo. 